\title{
Stability of the Closed Einstein Universe in Energy-Momentum Squared Gravity
}

\author{
M. Sharif *and M. Zeeshan Gul ${ }^{\dagger}$ \\ Department of Mathematics, University of the Punjab, \\ Quaid-e-Azam Campus, Lahore-54590, Pakistan.
}

\begin{abstract}
This paper analyzes the stability of the closed Einstein static universe by using linear homogeneous perturbations in the framework of energy-momentum squared gravity. This newly developed proposal resolves the primordial singularity and yields feasible cosmological results in the early times. For this purpose, we consider the closed Friedmann-Robertson-Walker universe with isotropic matter configuration and adopt small perturbations on the matter parameters and scale factor. Further, we establish equations of motion for static as well as perturbed systems and analyze the stable regions for particular $f\left(\mathcal{R}, \mathbf{T}^{2}\right)$ models corresponding to both conserved and non-conserved energy-momentum tensor. The graphical interpretation demonstrates that stable regions of the Einstein cosmos exist for all values of the model parameters and equation of state variable. We conclude that stable regions in this modified theory are large as compared to other modified theories of gravity.
\end{abstract}

Keywords: $f\left(\mathcal{R}, \mathbf{T}^{2}\right)$ Gravity; Einstein Universe; Stability Analysis. PACS: 04.50.Kd; 04.40.Dg; 04.25.Nx.

*msharif.math@pu.edu.pk

${ }^{\dagger}$ mzeeshangul.math@gmail.com 


\section{Introduction}

The current cosmic acceleration has been the most stunning and dazzling consequence for cosmologists over the past two decades. Scientists claim that this acceleration is caused by an ambiguous force dubbed as dark energy which has repulsive effects. This mysterious force has motivated numerous researchers to reveal its unknown attributes. In this framework, alternative gravitational theories are regarded as the most important and optimistic proposals to unveil the cryptic nature of dark components. These theories can be developed by adding scalar invariants and their corresponding functions in the geometric section of the Einstein-Hilbert action. The simplest modification of general relativity (GR) is $f(\mathcal{R})$ theory, where $\mathcal{R}$ is the Ricci scalar. A comprehensive literature [1] has been available to comprehend the viable aspects of this theory.

The interactions among curvature and matter parts generalized $f(\mathcal{R})$ theory. Such interactions determine the rotation curves of galaxies as well as different cosmic stages. These theories are non-conserved that provide the presence of an extra force. These coupling proposals are very useful to comprehend the cosmic acceleration and cryptic nature of dark components. Harko et al. [2] introduced such interactions in $f(\mathcal{R})$ theory named $f(\mathcal{R}, \mathcal{T})$ gravity, where $\mathcal{T}$ is the trace of the energy-momentum tensor (EMT). The non-minimal interaction between geometry and matter was developed in [3] named $f\left(\mathcal{R}, \mathcal{T}, \mathcal{R}_{\alpha \beta} \mathcal{T}^{\alpha \beta}\right)$ theory. One of the modification gives rise to $f\left(\mathbb{R}, \mathbb{T}^{\phi}\right)$ theory, where $\phi$ defines the scalar field [4].

The presence of singularities is considered a major problem in GR because of its prediction at the high energy level, where GR is no longer valid due to the expected quantum impacts. However, there is no specific formalism for quantum gravity. In this regard, a new covariant generalization of GR is developed by adding the analytic function $\mathcal{T}^{\alpha \beta} \mathcal{T}_{\alpha \beta}$ in the generic action known as energy-momentum squared gravity (EMSG). This is also referred to as $f\left(\mathcal{R}, \mathbf{T}^{2}\right)$ theory, where $\mathbf{T}^{2}$ is denoted by $\mathcal{T}^{\alpha \beta} \mathcal{T}_{\alpha \beta}$ [5]. This modification of GR is considered the most favorable and prosperous technique which resolves the spacetime singularity in the non-quantum description. Consequently, the corresponding field equations are different from GR only in the presence of matter sources. It contributes squared terms in the field equations that are used to explore various fascinating cosmological consequences.

Board and Barrow [6] studied the exact solutions of isotropic spacetime and investigated their behavior with cosmic growth, presence, and absence 
of singularities. Nari and Roshan [7] explored the feasible and stable compact stars in this context. Bahamonde et al. 8] analyzed the minimal and non-minimal EMSG models and concluded that these models determine the cosmic acceleration. Barbar et al [9] investigated physically viable conditions of the bouncing universe corresponding to a specific EMSG model. Rudra and Pourhassan 10] examined thermodynamic characteristics of a black hole. Singh et al [1] analyzed the geometry of compact objects in the existence of quark matter. Recently, we have discussed the Noether symmetry approach in this framework and investigated the viable behavior of cosmological solutions through different cosmological parameters. We have also studied the dynamics and stability of dense objects [12. It is found that modified EMSG terms boost the system's stability and hence prevent the collapsing phenomenon. The above literature makes it clear that this theory needs more focus and thus motivation to explore this theory further is very much obvious.

It has been found that at early times, all matter was concentrated to an infinitely compact point named primordial or big-bang singularity. This singularity is considered a major problem in cosmology. In this perspective, the emergent cosmic scenario is identified as a useful technique that helps to resolve the primordial singularity. According to this strategy, the cosmos initiates from the static state then progresses to the expansion process which causes the inflationary phase. In this context, the early state of the cosmos is the Einstein universe (EU) rather than primordial singularity. The presence, as well as stability of the EU against all sorts of perturbations, justify the emergent cosmos. Nevertheless, the concept of an emergent universe is not well illustrated in GR due to the existence of unstable solutions. Harrison [13] examined the stable regions of the EU through inhomogeneous perturbations in the presence of dust matter configuration. It was examined that stable regions exist if $5 c_{s}^{2}>1$ is satisfied, where $c_{s}$ is the speed of sound [14]. Barrow and Yamamoto [15] examined the stability of EU corresponding to various matter configurations and obtained the unstable regions against homogeneous perturbations.

Alternative gravitational theories have attained much attention to find stable solutions for the EU. The stable solutions of the EU employing homogeneous perturbations with specific $f(\mathcal{R})$ models have been analyzed in [16]. In this framework, the stable regions exist by homogenous perturbations whereas these stable modes become unstable corresponding to inhomogeneous perturbations. The stable modes of $\mathrm{EU}$ in $f(G)$ theory against homoge- 
neous perturbations has been examined in [17]. The stable regions of the EU by using scalar perturbations in modified teleparallel theory have been discussed in [18]. They found that stable EU appears for both open and closed universe models. The stable regimes in the modified Gauss-Bonnet theory against homogenous and inhomogeneous perturbations have been studied in [19]. The stability of EU in scalar-field theories has been investigated in [20] and found the stable as well as unstable regions corresponding to homogeneous and inhomogeneous perturbations.

The analysis of stable EU has also been a source of fascinating results in the context of curvature-matter interactions. The stability of EU in $f(\mathcal{R}, \mathcal{T})$ framework has been explored in [21] and found stable modes which were unstable in $f(\mathcal{R})$ theory. The stable regions of EU corresponding to both (homogeneous/inhomogeneous) perturbations in $f(G, \mathcal{T})$ theory has been analyzed in [22]. The stable modes of EU by using different perturbation techniques in $f\left(\mathcal{R}, \mathcal{T}, \mathcal{R}_{\alpha \beta} \mathcal{T}^{\alpha \beta}\right)$ background has been discussed in [23]. They also studied the stability criteria with inhomogeneous perturbations in $f(\mathcal{R}, \mathcal{T})$ gravity. The stable modes of EU with anisotropic homogeneous perturbations in curvature-matter coupled theory have been examined in [24].

In this paper, we use the homogeneous perturbations technique to examine the stability of closed EU in EMSG. This analysis will help to examine the impacts of curvature-matter interactions on the stability of the closed EU. The paper is organized as follows. We derive the equations of motion in the background of the closed FRW universe in section 2 . Section 3 investigates the stable regions of the closed EU for conserved and non-conserved EMT. A brief description and discussion of the outcomes are provided in the last section.

\section{Einstein Static Universe}

This section formulates the equations of motion for closed FRW spacetime in the context of $f\left(\mathcal{R}, \mathbf{T}^{2}\right)$ theory. The action for this gravity is [5]

$$
\mathcal{S}=\frac{1}{2 \kappa^{2}} \int f\left(\mathcal{R}, \mathbf{T}^{2}\right) \sqrt{-g} d^{4} x+\int \mathcal{L}_{M} \sqrt{-g} d^{4} x
$$

where the coupling constant, determinant of the line element and matter Lagrangian density is denoted by $\kappa^{2}, g$ and $\mathcal{L}_{M}$, respectively. This theory has maximum energy density and correspondingly a minimum scale factor at the 
early universe which indicates that there is a bounce at early times. Moreover, this theory possesses a true sequence of cosmological eras. Although the cosmological constant does not play a crucial role in the background of the standard cosmological model, however, the cosmological constant supports resolving singularity only after the matter-dominated era in EMSG. However, the profile of density supports the inflationary cosmological models that successfully provide convincing answers to major cosmological issues like horizon problem, flatness problem, etc. It is worthwhile to mention here that this theory overcomes the spacetime singularity but does not change the cosmological evolution. It is assumed that some useful results will be obtained to study the stability of the closed EU due to the matter-dominated era. The following equations of motion are obtained from the variation of action corresponding to the metric tensor.

$$
\mathcal{R}_{\alpha \beta} f_{\mathcal{R}}+g_{\alpha \beta} \square f_{\mathcal{R}}-\nabla_{\alpha} \nabla_{\beta} f_{\mathcal{R}}-\frac{1}{2} g_{\alpha \beta} f=\mathcal{T}_{\alpha \beta}^{(M)}-\Theta_{\alpha \beta} f_{\mathbf{T}^{2}},
$$

where $\square=\nabla_{\mu} \nabla^{\mu}, f \equiv f\left(\mathcal{R}, \mathbf{T}^{2}\right), f_{\mathbf{T}^{2}}=\frac{\partial f}{\partial \mathbf{T}^{2}}, f_{\mathcal{R}}=\frac{\partial f}{\partial \mathcal{R}}$,

$$
\Theta_{\alpha \beta}=-2 \mathcal{L}_{m}\left(\mathcal{T}_{\alpha \beta}-\frac{1}{2} g_{\alpha \beta} \mathcal{T}\right)-4 \frac{\partial^{2} \mathcal{L}_{M}}{\partial g^{\alpha \beta} \partial g^{\mu \nu}} \mathcal{T}^{\mu \nu}-\mathcal{T} \mathcal{T}_{\alpha \beta}+2 \mathcal{T}_{\alpha}^{\mu} \mathcal{T}_{\beta \mu}
$$

This theory reduces to $f(\mathcal{R})$ gravity for $f\left(\mathcal{R}, \mathbf{T}^{2}\right)=f(\mathcal{R})$ and to GR when $f\left(\mathcal{R}, \mathbf{T}^{2}\right)=\mathcal{R}$. The stress-energy tensor demonstrates the configuration of matter and energy in gravitational physics, whereas every non-zero component provides dynamical parameters with specific physical properties.

Here, we take into account isotropic matter configuration as

$$
\mathcal{T}_{\alpha \beta}^{(M)}=(\rho+\mathrm{p}) \mathcal{U}_{\alpha} \mathcal{U}_{\beta}-\mathrm{p} g_{\alpha \beta}
$$

where four-velocity, matter pressure and density are defined by $\mathcal{U}_{\alpha}, \mathrm{p}$ and $\rho$, respectively. Manipulating Eq.(3), we obtain

$$
\Theta_{\alpha \beta}=-\left(3 \mathrm{p}^{2}+\rho^{2}+4 \mathrm{p} \rho\right) \mathcal{U}_{\alpha} \mathcal{U}_{\beta} .
$$

Rearranging Eq.(21), we have

$$
\mathcal{G}_{\alpha \beta}=\frac{1}{f_{\mathcal{R}}}\left(\mathcal{T}_{\alpha \beta}^{(M)}+\mathcal{T}_{\alpha \beta}^{(D)}\right)
$$


where $\mathcal{G}_{\alpha \beta}$ is the Einstein tensor and $\mathcal{T}_{\alpha \beta}^{(D)}$ are the additional impacts of EMSG that include the higher-order curvature terms because of the modification in curvature part named dark source terms expressed as

$$
\mathcal{T}_{\alpha \beta}^{(D)}=\frac{1}{2} g_{\alpha \beta}\left(f-\mathcal{R} f_{\mathcal{R}}\right)-\Theta_{\alpha \beta} f_{\mathbf{T}^{2}}+\left(\nabla_{\alpha} \nabla_{\beta}-g_{\alpha \beta} \square\right) f_{\mathcal{R}}
$$

The $f\left(\mathcal{R}, \mathbf{T}^{2}\right)$ gravity provides non-conserved EMT implying the presence of an extra force that acts as a non-geodesic motion of particles given by

$$
\nabla^{\alpha} T_{\alpha \beta}^{(M)}=-\frac{1}{2}\left(f_{\mathbf{T}^{2}} g_{\alpha \beta} \nabla^{\alpha} \mathbf{T}^{2}-2 \nabla^{\alpha}\left(f_{\mathbf{T}^{2}} \Theta_{\alpha \beta}\right)\right) .
$$

In order to study the isotopic homogenous universe, we consider closed FRW spacetime as [16]

$$
d s^{2}=d \mathrm{t}^{2}-\frac{\mathrm{a}^{2}(\mathrm{t})}{1-\mathrm{r}^{2}} d \mathrm{r}^{2}-\mathrm{a}^{2}(\mathrm{t}) \mathrm{r}^{2} d \theta^{2}-\mathrm{a}^{2}(\mathrm{t}) \mathrm{r}^{2} \sin ^{2} \theta d \phi^{2}
$$

where $\mathrm{a}(\mathrm{t})$ is the scale parameter. The corresponding field equations become

$$
\begin{aligned}
\frac{3}{\mathrm{a}^{2}}\left(1+\dot{\mathrm{a}}^{2}\right) & =\frac{1}{f_{\mathcal{R}}}\left\{\rho+\frac{1}{2} f\left(\mathcal{R}, \mathbf{T}^{2}\right)+3\left(\frac{1+\dot{\mathrm{a}}^{2}+\mathrm{a} \ddot{a}}{\mathrm{a}^{2}}\right) f_{\mathcal{R}}\right. \\
& \left.-\frac{3 \dot{\mathrm{a}}}{\mathrm{a}} \dot{f}_{\mathcal{R}}+\left(3 \mathrm{p}^{2}+\rho^{2}+4 \mathrm{p} \rho\right) f_{\mathbf{T}^{2}}\right\}, \\
-2 \mathrm{a} \ddot{\mathrm{a}}-\left(1+\dot{\mathrm{a}}^{2}\right) & =\frac{1}{f_{\mathcal{R}}}\left\{\mathrm{a}^{2} \mathrm{p}-\frac{\mathrm{a}^{2}}{2} f\left(\mathcal{R}, \mathbf{T}^{2}\right)-3\left(1+\dot{\mathrm{a}}^{2}+\mathrm{a} \ddot{\mathrm{a}}\right) f_{\mathcal{R}}\right. \\
& \left.+2 \mathrm{a} \dot{\dot{f}_{\mathcal{R}}}+\mathrm{a}^{2} \ddot{f_{\mathcal{R}}}\right\} .
\end{aligned}
$$

These equations describe how gravity and fluid parameters bend spacetime. Here

$$
\mathcal{R}=-6\left(\frac{\mathrm{a} \ddot{\mathrm{a}}+\dot{\mathrm{a}}^{2}+1}{\mathrm{a}^{2}}\right), \quad \mathbf{T}^{2}=3 \mathrm{p}^{2}+\rho^{2},
$$

where dot demonstrates the derivative corresponding to temporal coordinate. The non-conservation equation for perfect fluid turns out to be

$$
\begin{aligned}
\dot{\rho}+\frac{3 \dot{\mathrm{a}}}{\mathrm{a}}(\rho+\mathrm{p}) & =\left(3 \mathrm{p}^{2}+\rho^{2}+4 \mathrm{p} \rho\right) \dot{f}_{\mathbf{T}^{2}}-\frac{3 \dot{\mathrm{a}}}{\mathrm{a}}\left(3 \mathrm{p}^{2}+\rho^{2}+4 \mathrm{p} \rho\right) f_{\mathbf{T}^{2}} \\
& -f_{\mathbf{T}^{2}}\{\dot{\rho}(3 \rho+4 \mathrm{p})+\dot{\mathrm{p}}(9 \mathrm{p}+4 \rho)\} .
\end{aligned}
$$




\section{$3 \quad$ Stability Analysis}

In this section, we examine the stability of closed EU by using homogenous perturbations in EMSG. For this reason, the resulting equations of motion minimize to

$$
\begin{aligned}
\frac{3}{\mathrm{a}_{0}^{2}} & =\frac{1}{f_{\mathcal{R}}}\left\{\rho_{0}+\frac{1}{2} f\left(\mathcal{R}_{0}, \mathbf{T}_{0}^{2}\right)+\frac{3}{\mathrm{a}_{0}^{2}} f_{\mathcal{R}}+\left(3 \mathrm{p}_{0}^{2}+\rho_{0}^{2}+4 \mathrm{p}_{0} \rho_{0}\right) f_{\mathbf{T}^{2}}\right\}, \\
-\frac{1}{\mathrm{a}_{0}^{2}} & =\frac{1}{f_{\mathcal{R}}}\left\{\mathrm{p}_{0}-\frac{1}{2} f\left(\mathcal{R}_{0}, \mathbf{T}_{0}^{2}\right)-\frac{3}{\mathrm{a}_{0}^{2}} f_{\mathcal{R}}+\ddot{f}_{\mathcal{R}}\right\},
\end{aligned}
$$

where $\mathrm{a}_{0}=$ constant, $\mathcal{R}_{0}=\frac{6}{\mathrm{a}_{0}^{2}}=\mathcal{R}\left(\mathrm{a}_{0}\right)$ and $\mathbf{T}_{0}^{2}=\rho_{0}^{2}+3 \mathrm{p}_{0}^{2}$. Here $\rho_{0}$ and $\mathrm{p}_{0}$ represent the unperturbed matter variables. We take linear $\operatorname{EoS}(p=\omega \rho)$ to examine the stable regions of closed EU and determine the homogeneous perturbations in matter density and scale parameter defined as

$$
\rho(\mathrm{t})=(\delta \rho(\mathrm{t})+1) \rho_{0}, \quad \mathrm{a}(\mathrm{t})=(\delta \mathrm{a}(\mathrm{t})+1) \mathrm{a}_{0},
$$

where perturbed matter density and scale parameter are represented by $\delta \rho(\mathrm{t})$ and $\delta \mathrm{a}(\mathrm{t})$, respectively. By using Taylor series expansion upto first order and consider $f\left(\mathcal{R}, \mathbf{T}^{2}\right)$ as an analytic function, we have

$$
f\left(\mathcal{R}, \mathbf{T}^{2}\right)=f\left(\mathcal{R}_{0}, \mathbf{T}_{0}^{2}\right)+f\left(\mathcal{R}_{0}, \mathbf{T}_{0}^{2}\right) \delta \mathcal{R}+f\left(\mathcal{R}_{0}, \mathbf{T}_{0}^{2}\right) \delta \mathbf{T}^{2},
$$

where $\delta \mathcal{R}$ and $\delta \mathbf{T}^{2}$ have the following relations

$$
\delta \mathcal{R}=-6\left(\delta \ddot{\mathrm{a}}-\frac{2 \delta \mathrm{a}}{\mathrm{a}_{0}^{2}}\right), \quad \delta \mathbf{T}^{2}=\rho_{0}^{2}\left(3 \omega^{2}+1\right) \delta \rho .
$$

Here $\delta \ddot{\mathrm{a}}(\mathrm{t})=\frac{d^{2}}{d \mathrm{t}^{2}}(\delta \mathrm{a}(\mathrm{t}))$. Using Eqs.(8) $-(15)$, the linearized perturbed equations of motion turn out to be

$$
\begin{aligned}
& 6 f_{\mathcal{R}} \delta \mathrm{a}+\mathrm{a}_{0}^{2} \rho_{0}\left\{1+\frac{\rho_{0}}{2}\left(15 \omega^{2}+16 \omega+5\right) f_{\mathbf{T}^{2}}\right\} \delta \rho=0 \\
& 2\left(\delta \ddot{\mathrm{a}}-\frac{1}{\mathrm{a}_{0}^{2}} \delta \mathrm{a}\right) f_{\mathcal{R}}+\rho_{0}\left\{\omega-\frac{1}{2} \rho_{0}\left(1+3 \omega^{2}\right) f_{\mathbf{T}^{2}}\right\} \delta \rho=0 .
\end{aligned}
$$

These equations determine the direct relation between perturbed matter density and scale factor.

The following sections address the stability of the closed EU for vanishing and non-vanishing divergence of EMT. 


\subsection{Stability for Conserved EMT}

It is well-known that curvature-matter coupled theories are non-conserved. Here, we assume that the general conservation law satisfies in EMSG and hence right side of Eq.(10) must be zero which yields

$$
\begin{aligned}
& f_{\mathbf{T}^{2}}\{\dot{\rho}(3 \rho+4 \mathrm{p})+\dot{\mathrm{p}}(9 \mathrm{p}+4 \rho)\}+\frac{3 \dot{\mathrm{a}}}{\mathrm{a}}\left(3 \mathrm{p}^{2}+\rho^{2}+4 \mathrm{p} \rho\right) f_{\mathbf{T}^{2}} \\
& -\left(3 \mathrm{p}^{2}+\rho^{2}+4 \mathrm{p} \rho\right) \dot{f}_{\mathbf{T}^{2}}=0 .
\end{aligned}
$$

We use Eqs.(16) and (17) to obtain the perturbed equation of motion in terms of perturbed scale factor as follows

$$
\begin{aligned}
& \delta \mathrm{a}\left\{3 \omega+1+\frac{\rho_{0}}{2}\left(6 \omega^{2}+16 \omega+2\right) f_{\mathbf{T}^{2}}\right\}-\delta \ddot{\mathrm{a}} \\
& \times \mathrm{a}_{0}^{2}\left\{1+\frac{\rho_{0}}{2}\left(15 \omega^{2}+16 \omega+5\right) f_{\mathbf{T}^{2}}\right\}=0 .
\end{aligned}
$$

Manipulating Eqs.(11) and (12), we have

$$
\mathrm{a}_{0}^{2}=\frac{2 f_{\mathcal{R}}}{\rho_{0}(1+\omega)+\rho_{0}^{2}\left(3 \omega^{2}+4 \omega+1\right) f_{\mathbf{T}^{2}}} .
$$

Using this value in Eqs.(19), the perturbed equation of motion becomes

$$
\begin{aligned}
& \delta \mathrm{a}\left\{3 \omega+1+\frac{\rho_{0}}{2}\left(6 \omega^{2}+16 \omega+2\right) f_{\mathbf{T}^{2}}\right\}\left\{\rho_{0}^{2}\left(3 \omega^{2}+4 \omega+1\right) f_{\mathbf{T}^{2}}\right. \\
& \left.+\rho_{0}(1+\omega)\right\}-f_{\mathcal{R}}\left\{2+\rho_{0}\left(15 \omega^{2}+16 \omega+5\right) f_{\mathbf{T}^{2}}\right\} \delta \ddot{\mathrm{a}}=0 .
\end{aligned}
$$

We also have fourth-order perturbed equations of motion as appearing in other modified theories but only first-order linear terms appear due to the existence of $\mathbf{T}^{2}$ term. Therefore, the second-order perturbed field equation is obtained about the scale factor in this gravity. Equation (21) minimizes to the following desired form in the GR limits $\left(f_{\mathcal{R}}=1\right.$ and $\left.f_{\mathbf{T}^{2}}=0\right)$ as

$$
2 \delta \ddot{\mathrm{a}}-\rho\left(3 \omega^{2}+4 \omega+1\right) \delta \mathrm{a}=0 .
$$

The solution of the perturbed field equation (21) helps to investigate the stable regions of the closed Einstein universe but this equation is quite complicated due to the presence of multivariate functions and their derivatives.

To explore the viability of this result in EMSG, we consider a specific type of generic function which gives minimal and non-minimal couplings between 
geometric and matter parts. The non-minimal model, $f\left(\mathcal{R}, \mathbf{T}^{2}\right)=f_{0} \mathcal{R} \mathbf{T}^{2}$, yields the perturbed field equation in a complex form and we are unable to conclude any outcome from this model. So, for the sake of convenience, we take the minimal coupling model as [5]

$$
f\left(\mathcal{R}, \mathbf{T}^{2}\right)=f_{1}(\mathcal{R})+f_{2}\left(\mathbf{T}^{2}\right) .
$$

This model represents an interesting cosmological model which can explain the current evolution of the universe and the emergence of the accelerated expansion as a geometrical consequence. We have considered conserved EMT, hence the conservation law satisfies this model.

Manipulating Eq.(18), we obtain

$$
f_{2}^{\prime}\left(\mathbf{T}^{2}\right)\left(9 \omega^{2}+8 \omega+3\right)+f_{2}^{\prime \prime}\left(\mathbf{T}^{2}\right)\left(3 \omega^{2}+4 \omega+1\right)\left(6 \omega^{2}+2\right) \rho_{0}^{2}=0,
$$

where prime determines the derivative corresponding to $x$, i.e., $x=\mathcal{R}$ or $\mathbf{T}^{2}$. The solution of the above equation is given by

$$
f_{2}\left(\mathbf{T}^{2}\right)=b_{1} e^{\frac{-\left(9 \omega^{2}+8 \omega+3\right) \mathbf{T}^{2}}{2 \rho_{0}^{2}(\omega+1)(3 \omega+1)\left(3 \omega^{2}+1\right)}}+b_{2}
$$

where $b_{1}$ and $b_{2}$ are constants of integration. It should be noted that only this expression of $f\left(\mathbf{T}^{2}\right)$ satisfies the conservation law in the model (22). Now using the preceding equations, Eq.(21) turns out to be

$$
\left(\Delta_{1} \Delta_{3}-\Delta_{1} \Delta_{4} \Delta_{7}-\Delta_{2} \Delta_{3} \Delta_{7}+\Delta_{2} \Delta_{4} \Delta_{7}^{2}\right) \delta \mathrm{a}-f_{1}^{\prime}(\mathcal{R})\left(\Delta_{5}-\Delta_{6} \Delta_{7}\right) \delta \ddot{\mathrm{a}}=0,(24)
$$

where $\Delta_{i}^{\prime} s(i=1,2,3,4,5,6,7)$ are

$$
\begin{aligned}
& \Delta_{1}=4 \rho_{0}^{2}\left(9 \omega^{2}+6 \omega+1\right)(\omega+1)\left(3 \omega^{2}+1\right), \\
& \Delta_{2}=\rho_{0} b_{1}\left(6 \omega^{2}+16 \omega+2\right)\left(9 \omega^{2}+8 \omega+3\right), \\
& \Delta_{3}=2 \rho_{0}^{3}\left(\omega^{2}+2 \omega+1\right)(3 \omega+1)\left(3 \omega^{2}+1\right), \\
& \Delta_{4}=\rho_{0}^{2} b_{1}\left(3 \omega^{2}+4 \omega+1\right)\left(9 \omega^{2}+8 \omega+3\right), \\
& \Delta_{5}=8 \rho_{0}^{2}(\omega+1)(3 \omega+1)\left(3 \omega^{2}+1\right) \\
& \Delta_{6}=2 \rho_{0} b_{1}\left(15 \omega^{2}+16 \omega+5\right)\left(9 \omega^{2}+8 \omega+3\right), \\
& \Delta_{7}=e^{\frac{-\left(9 \omega^{2}+8 \omega+3\right)}{2 \rho_{0}^{2}(\omega+1)(3 \omega+1)\left(3 \omega^{2}+1\right)}} .
\end{aligned}
$$

Equation.(24) has the following solution

$$
\delta a(t)=c_{1} e^{\Omega t}+c_{2} e^{-\Omega t},
$$


where integration constants are represented by $c_{1}$ and $c_{2}$ and the factor $\Omega$ defines the frequency of small perturbation given by

$$
\Omega^{2}=\frac{\left(\Delta_{3}-\Delta_{4} \Delta_{7}\right)\left(\Delta_{1}-\Delta_{2} \Delta_{7}\right)}{f_{1}^{\prime}(\mathcal{R})\left(\Delta_{5}-\Delta_{6} \Delta_{7}\right)} .
$$

This parameter must be less than zero to prevent the exponential growth or collapse which yields stability of the closed EU. This frequency in GR limit is defined as

$$
\Omega^{2}=\frac{\rho_{0}}{2}\left(3 \omega^{2}+4 \omega+1\right),
$$

which yields stable modes in the interval $\omega \in\left(-1,-\frac{1}{3}\right)$ [17].

We consider $\rho_{0}=0.3$ [25] and $f_{1}^{\prime}(\mathcal{R})=\xi_{1}$ as a new parameter to examine the stable modes of closed EU graphically. Here, the red regions represent the stable modes of the closed EU. The graphical interpretation in Figure 1 determines the stable regions of closed EU corresponding to $\Omega^{2}$ against homogeneous perturbations for $b_{1}>0$. It is analyzed that stable regions are obtained for all values of $\omega$ and these stable modes become more smooth as the integration constant decreases. Figure $\mathbf{2}$ shows the stability of closed EU for negative values of $b_{1}$. It is examined that stable regions become more smooth corresponding to $\omega$ as the value of $b_{1}$ increases. It is clear from Figures $\mathbf{1}$ and $\mathbf{2}$ that we obtain the stable regions of closed EU which were not stable in other modified theories of gravity [22]-[24].

\subsection{Stability for Non-Conserved EMT}

This section analyzes stable regions of the closed EU for non-zero divergence of EMT. Equation (6) determines the general expression of non-conserved EMT, which comes because of the higher-order derivatives of EMT that naturally exist in the equations of motion. This appears to be a problem for all alternative theories of gravity that include the interaction between curvature and matter parts. We consider a specific EMSG model as [6]

$$
f\left(\mathcal{R}, \mathbf{T}^{2}\right)=f_{1}(\mathcal{R})+\chi\left(\mathbf{T}^{2}\right)^{n},
$$

to explore the impacts of non-zero divergence of EMT on the stability of closed EU. Here, $\chi$ is the arbitrary constant. The perturbed equations of motion for the considered model turn out to be

$$
\mathrm{a}_{0}^{2}\left\{\rho_{0}+\frac{\chi n}{2}\left(\rho_{0}^{2}\left(3 \omega^{2}+1\right)\right)^{n-1}\left(15 \omega^{2}+16 \omega+5\right) \rho_{0}^{2}\right\} \delta \rho
$$



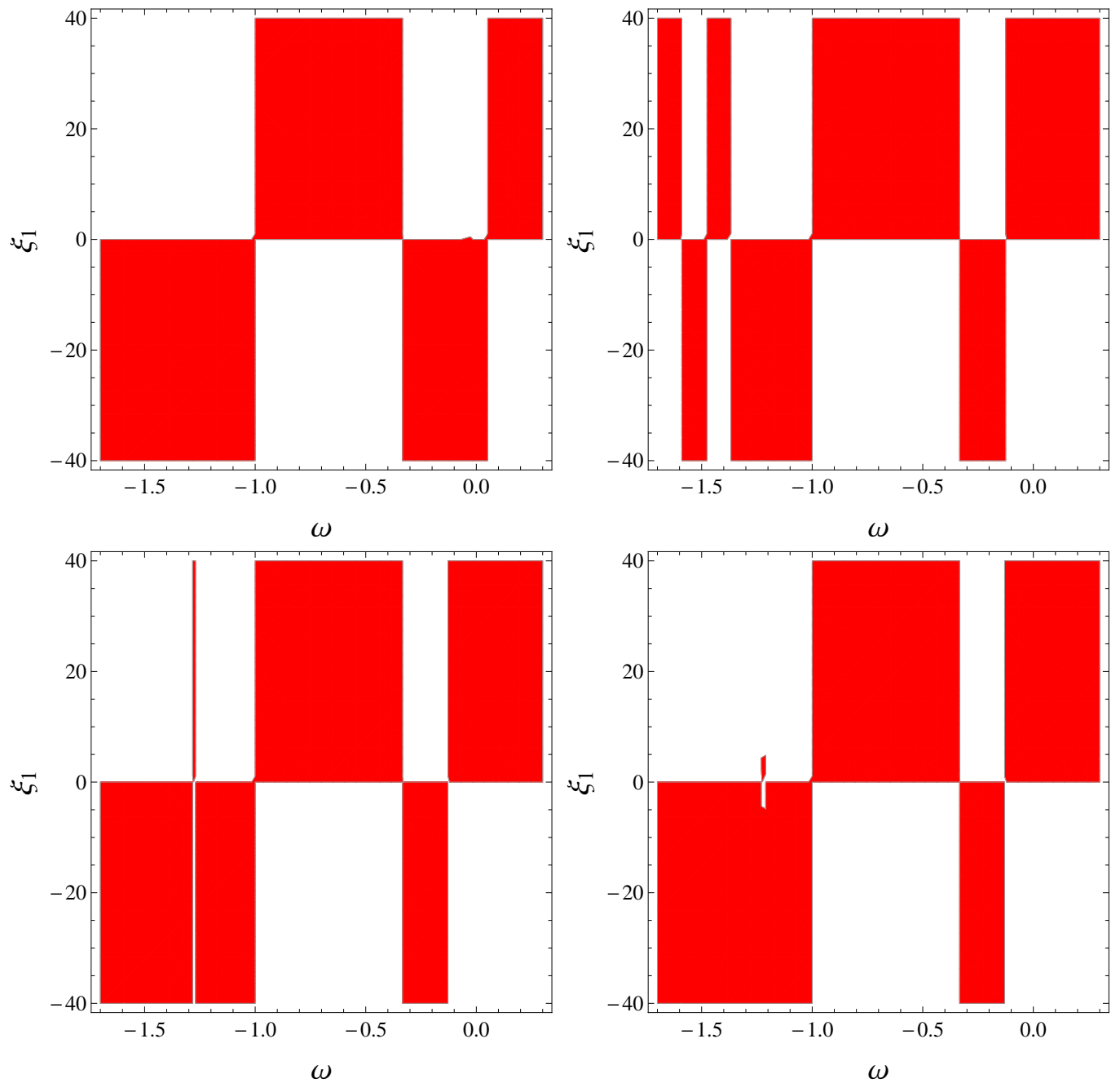

Figure 1: Stable modes in $\left(\omega, \xi_{1}\right)$ space corresponding to $b_{1}=1,15,30$ and 60 from left to right. 

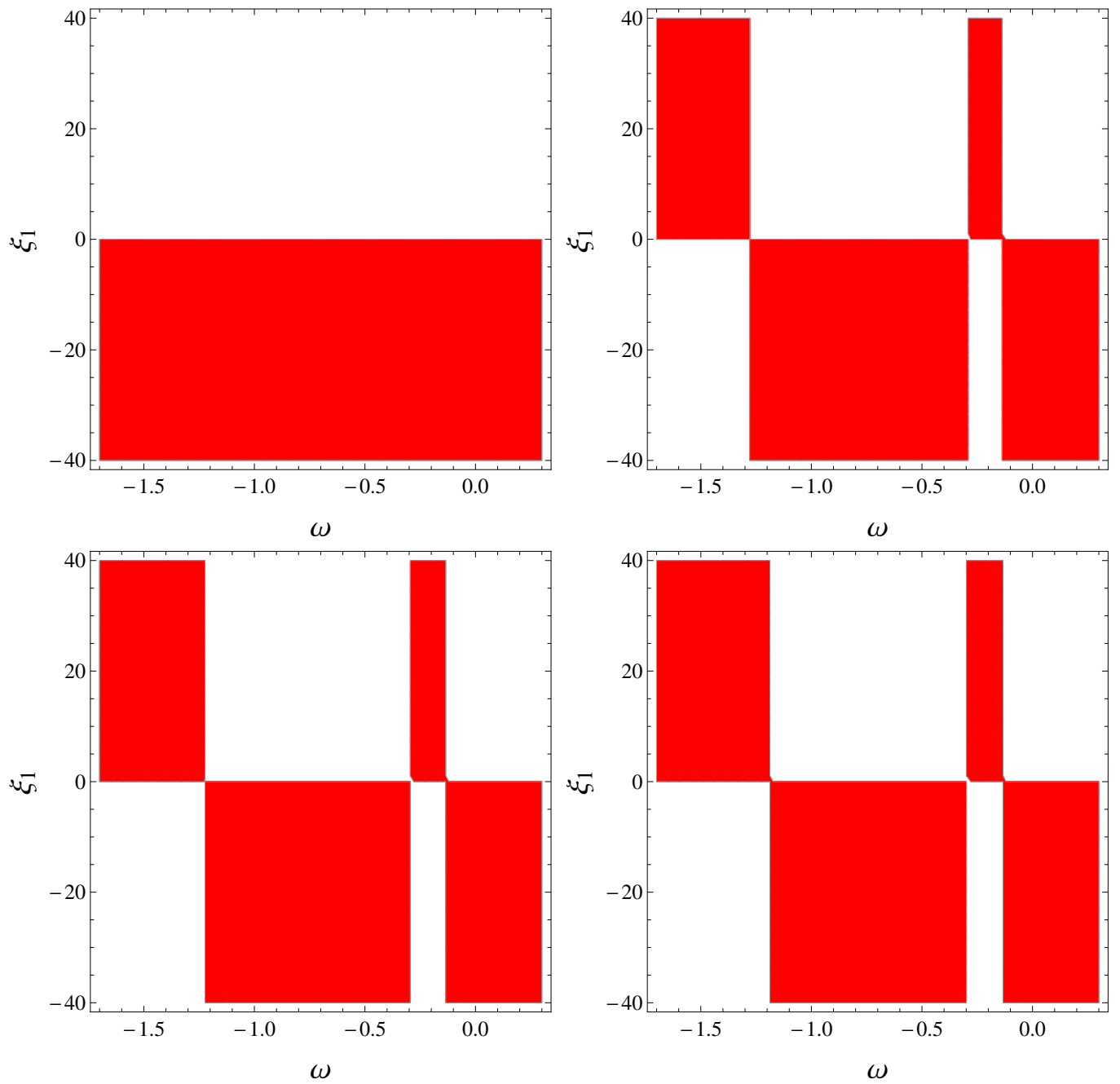

Figure 2: Stable modes in $\left(\omega, \xi_{1}\right)$ space corresponding to $b_{1}=-1,-15,-30$ and -60 from left to right. 


$$
\begin{aligned}
& +6 f_{1}^{\prime}(\mathcal{R}) \delta \mathrm{a}=0, \\
& \left\{\rho_{0} \omega-\frac{\chi n}{2}\left(\rho_{0}^{2}\left(3 \omega^{2}+1\right)\right)^{n-1}\left(1+3 \omega^{2}\right) \rho_{0}^{2}\right\} \delta \rho \\
& +2\left(\delta \ddot{\mathrm{a}}-\frac{1}{\mathrm{a}_{0}^{2}} \delta \mathrm{a}\right) f_{1}^{\prime}(\mathcal{R})=0 .
\end{aligned}
$$

These equations determine the correlation between perturbed matter density as well as the scale factor. The perturbed equation of motion in terms of perturbed scale parameter becomes

$$
\begin{aligned}
& \delta \mathrm{a}\left\{3 \omega+1+\frac{\chi n}{2}\left(\rho_{0}^{2}\left(3 \omega^{2}+1\right)\right)^{n-1}\left(6 \omega^{2}+16 \omega+2\right) \rho_{0}\right\}-\delta \ddot{\mathrm{a}} \\
& \times \mathrm{a}_{0}^{2}\left\{1+\frac{\chi n}{2}\left(\rho_{0}^{2}\left(3 \omega^{2}+1\right)\right)^{n-1}\left(15 \omega^{2}+16 \omega+5\right) \rho_{0}\right\}=0 .
\end{aligned}
$$

The addition of Eqs.(11) and (12) with respect to the model (26) gives

$$
a_{0}^{2}=2 f_{1}^{\prime}(\mathcal{R})\left\{\rho_{0}\left((1+\omega)+n \chi\left(\rho_{0}^{2}\left(3 \omega^{2}+1\right)\right)^{n-1}\left(3 \omega^{2}+4 \omega+1\right) \rho_{0}^{2}\right)\right\}^{-1} .
$$

Substituting this value in Eq.(29), the corresponding differential equation becomes

$$
\begin{aligned}
& \delta \mathrm{a}\left(\Delta_{1}+\rho_{0}^{2 n-2} \chi n \Delta_{2}\right)\left(\Delta_{3}+\rho_{0}^{2 n-2} \chi n \Delta_{4}\right)-f_{1}^{\prime}(\mathcal{R}) \rho_{0} \delta \ddot{\mathrm{a}} \\
& \left(\frac{2}{\rho_{0}}+\rho_{0}^{2 n-2} \chi n \Delta_{5}\right)=0
\end{aligned}
$$

where $\Delta_{i}^{\prime} s(i=1,2,3,4)$ are

$$
\begin{aligned}
& \Delta_{1}=3 \omega+1, \\
& \Delta_{2}=\frac{\rho_{0}}{2}\left(6 \omega^{2}+16 \omega+2\right)\left(\left(3 \omega^{2}+1\right)\right)^{n-1}, \\
& \Delta_{3}=\rho_{0}(1+\omega) \\
& \Delta_{4}=\rho_{0}^{2}\left(3 \omega^{2}+4 \omega+1\right)\left(\left(3 \omega^{2}+1\right)\right)^{n-1}, \\
& \Delta_{5}=\left(15 \omega^{2}+16 \omega+5\right)\left(\left(3 \omega^{2}+1\right)\right)^{n-1} .
\end{aligned}
$$

Equation (30) yields the following solution

$$
\delta \mathrm{a}(\mathrm{t})=d_{1} e^{\Omega t}+d_{2} e^{-\Omega \mathrm{t}},
$$



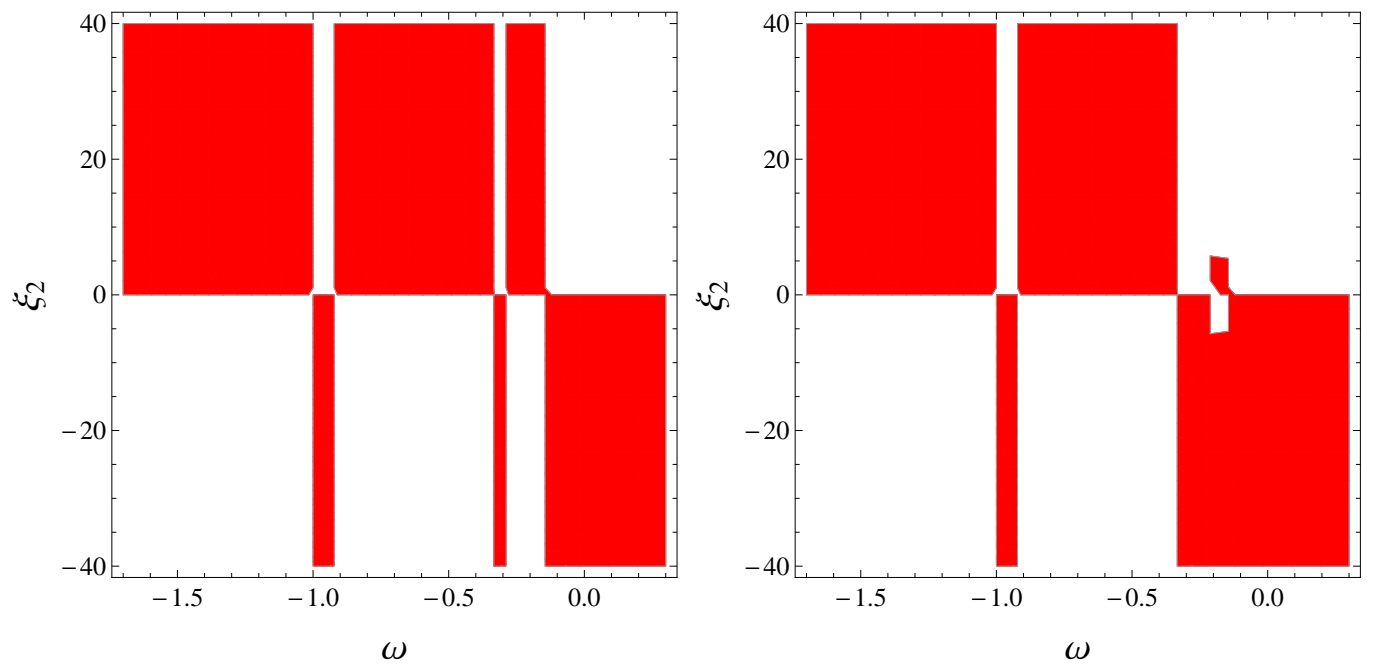

Figure 3: Stable modes in $\left(\omega, \xi_{2}\right)$ space corresponding to $\chi=1,5$ from left to right.

where

$$
\Omega^{2}=\frac{\Delta_{4} \rho_{0} n \chi\left(\Delta_{1}+\Delta_{2} \chi n \rho_{0}^{n}\right) \Delta_{3} \rho_{0}\left(\Delta_{2} \chi n+\Delta_{1} \rho_{0}^{-2(n-1)}\right)}{\xi_{2} \Delta_{5} \chi n}
$$

Here $\xi_{2}=\rho_{0} f_{1}^{\prime}(\mathcal{R})$.

The graphical behavior of closed EU stability for $n=1$ and different values of the model parameters is given in Figures $\mathbf{3}$ and $\mathbf{4}$. It is examined that stable modes appear for all values of the model parameters. Figures 5 and 6 show the stability of closed EU for $\chi=1$ and distinct values of $n$. We find that stable regions appear for all values of the EoS variable and these stable modes become more smooth as $n$ increases. It is observed that the obtained stable regions are unstable in other alternative gravitational theories.

\section{Final Remarks}

The stability of the EU is considered the most debatable problem in cosmology. Einstein tried to find a static solution of his field equations to describe the isotropic and homogeneous universe. Since the field equations of GR 

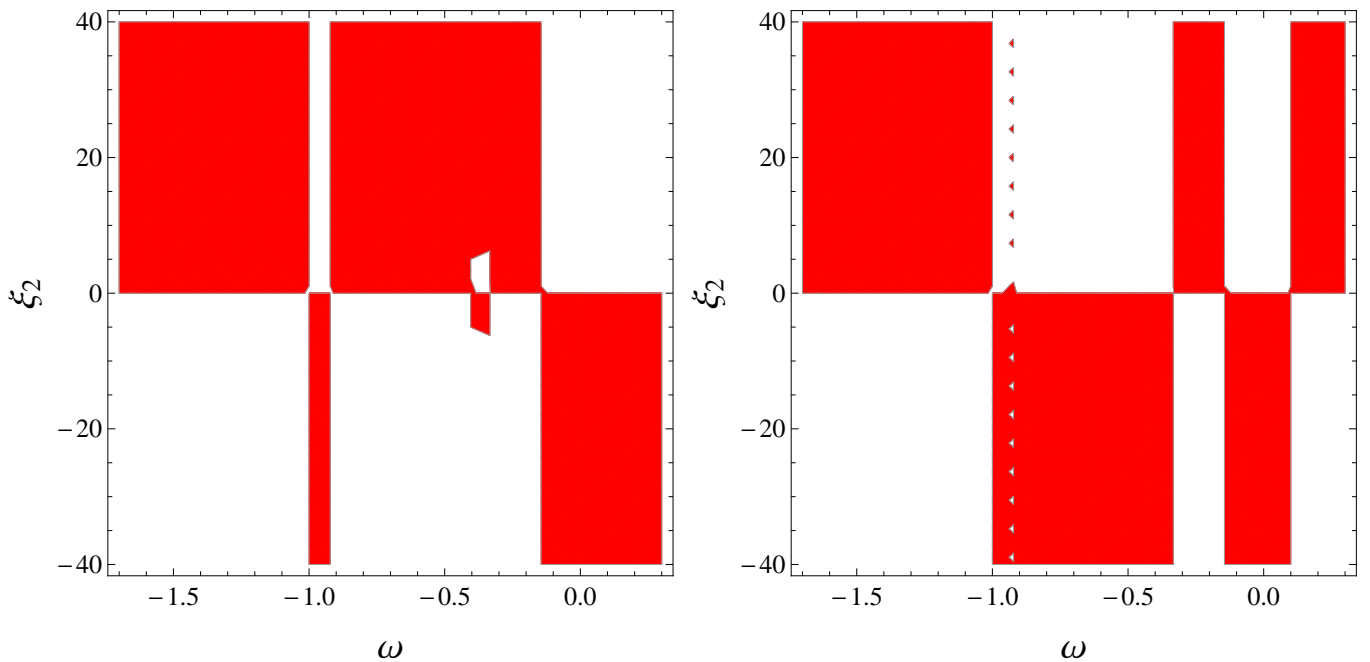

Figure 4: Stable regions in $\left(\omega, \xi_{2}\right)$ space corresponding to $\chi=-1,-5$ from left to right.
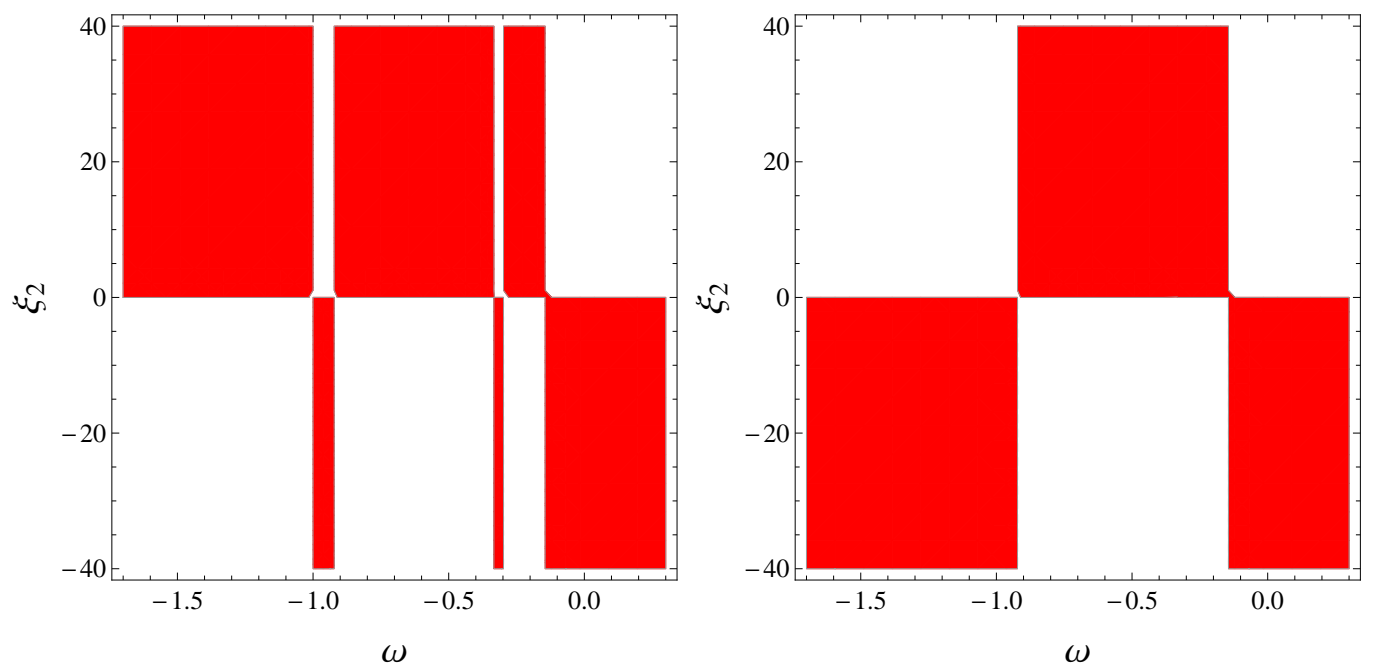

Figure 5: Stable modes in $\left(\omega, \xi_{2}\right)$ space corresponding to $n=0.5,5$ from left to right. 

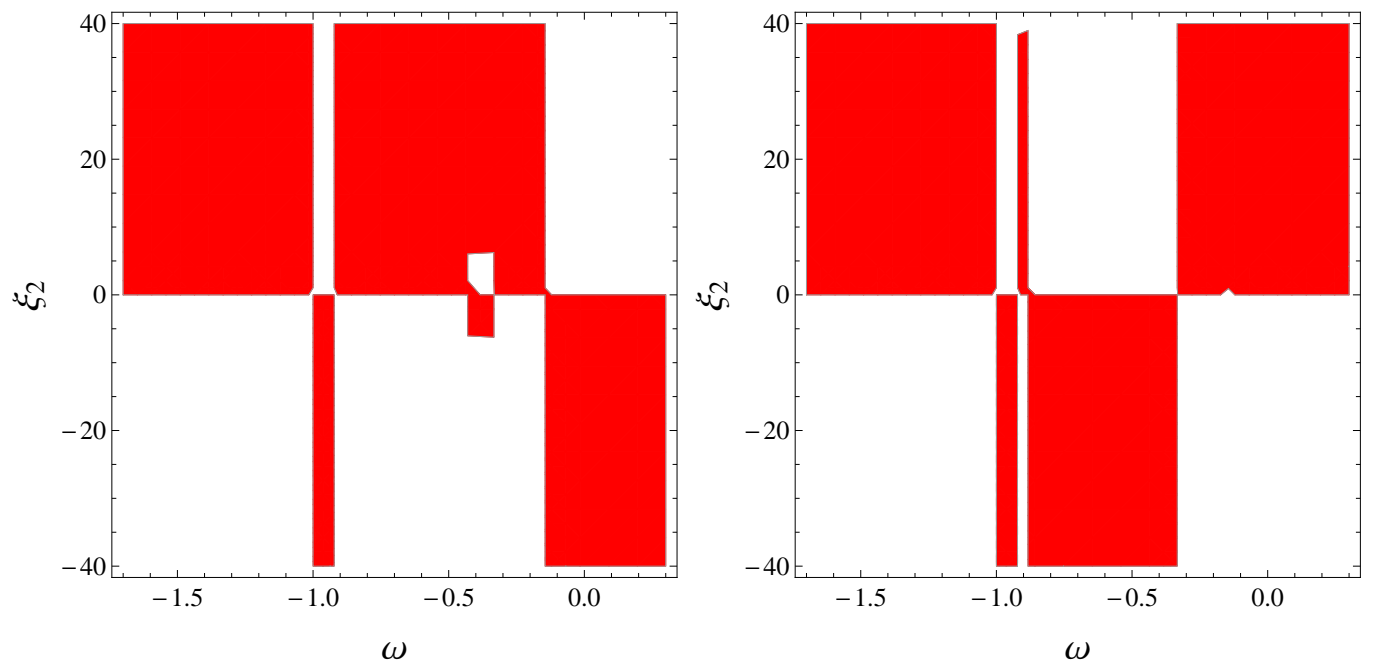

Figure 6: Stable regions in $\left(\omega, \xi_{2}\right)$ space corresponding to $n=-.05,-5$ from left to right.

have no static solution, therefore, Einstein introduced the term known as cosmological constant $(\Lambda)$ to have static solutions. It is important to know whether it can provide a natural initial state for a past eternal universe, whether it allows the universe to evolve away from this state, and whether under any circumstances it can act as an attractor for the very early evolution of the universe. With these questions in mind, we have investigated in detail that the closed EU is stable or unstable against linear homogeneous perturbations.

One of the most fundamental issues in cosmology is the mystery behind the beginning as well as the origin of the universe. According to some physical constraints on cosmic matter configuration, GR equations suggest that the current expanding cosmos must be anticipated by a singularity known as the big-bang singularity, where the physical parameters like energy density and spacetime curvature diverge. The emergent universe scenario is based on the pillars of the stable EU and is considered a favorable approach in cosmology to resolve the captivating issue of primordial singularity. The initial state of the universe in this framework is the EU instead of primordial singularity and then smoothly evolves to the rapid exponential inflationary era [26]. This conjecture implies that the initial cosmic epoch is the EU which enters into an expanding posture that leads to the inflationary phase. The phenomenon 
of EU is mainly manifested by the closed FLRW universe with isotropic fluid and the cosmological constant. The most essential characteristic of a successful emergent universe depends upon the stable solutions of the EU for any type of perturbation.

In this paper, we have investigated the stability of closed EU by using homogenous perturbations in EMSG. This newly proposed theory is nonconserved due to the interaction between curvature and matter parts. We have established equations of motion for the static and perturbed system through a linear EoS parameter. We have chosen specific $f\left(\mathcal{R}, \mathbf{T}^{2}\right)$ models to construct the perturbed differential equations whose solutions help to explore the stable regimes of closed EU. For the considered models, we have examined the conserved/non-conserved EMT cases against the homogeneous perturbations technique. The major results are given as follows.

- We have formulated a particular expression of $f_{2}\left(\mathbf{T}^{2}\right)$ for conserved EMT which fulfills the conservation equation. It is analyzed that stable regions of the closed EU exist for all choices of the integration constant.

- In the non-conserved case, we have considered a specific form of $f_{2}\left(\mathbf{T}^{2}\right)$ and examined the stability of closed EU for arbitrary values of the model parameters. It is found that the stable closed EU exists for all values of the model parameters.

We conclude that for all values of the EoS parameter, stable regions of closed EU appear in EMSG. Since all initial vector perturbations remain frozen, therefore, the stable modes by vector perturbations also appear for all EoS values.

It is worthwhile to mention here that the range of $\omega$ in EMSG increases and provides stable modes corresponding to all values of $\omega$ for both conserved as well as non-conserved EMT that are not stable in other modified theories. For example in $f(G, \mathcal{T})$ theory, it is found that for positive values of integration constant, stable EU exists for negative values of $\omega$ while no stable region exists for its positive values as well as negative values of integration constant in the conserved EMT case. For non-conserved EMT, stable regions are observed only for negative values of the model parameter and EoS parameter [27]. In $f(\mathcal{R}, \mathcal{T}, Q)$ theory, it is analyzed that for positive values of integration constant, the stable EU exists only for $\omega<1$ and stable modes exist in the range $-1<\omega<\frac{1}{3}$ for negative values of integration constant in the conserved EMT case. In the non-conserved EMT case, stability decreases 
with decreasing value of the model parameter [28]. It would be interesting to extend this work for inhomogeneous perturbations which indeed could yield a useful framework for the stability of closed EU in EMSG.

\section{References}

[1] Cognola, G. et al.: Phys. Rev. D 77(2008)046009; Felice, A.D. and Tsujikawa, S.R.: Living Rev. Relativ. 13(2010)3; Nojiri, S. and Odintsov, S.D.: Phys. Rep. 505(2011)59; Bamba, K. et al.: Astrophys. Space Sci. 342(2012)155.

[2] Harko, T. et al.: Phys. Rev. D 84(2011)024020.

[3] Haghani, Z. et al.: Phys. Rev. D 88(2013)044023.

[4] Moraes, P.H.R.S. and Santos, J.R.L.: Eur. Phys. J. C 76(2016)1.

[5] Katirci, N. and Kavuk, M.: Eur. Phys. J. Plus 129(2014)163.

[6] Board, C.V.R. and Barrow, J.D.: Phys. Rev. D 96(2017)123517.

[7] Moraes, P.H.R.S. and Sahoo, P.K.: Phys. Rev. D 97(2018)024007.

[8] Bahamonde, S., Marciu, M. and Rudra, P.: Phys. Rev. D 100(2019)083511.

[9] Barbar, A.H., Awad, A.M. and AlFiky, M.T.: Phys. Rev. D 101(2020)044058.

[10] Rudra, P. and Pourhassan, B.: arXiv:2008.11034v1.

[11] Singh, K.N. et al.: Phys. Dark Universe 31(2021)100774.

[12] Sharif, M.; Gul M.Z.: Phys. Scr. 96(2020)025002; Int. J. Mod. Phys. A 2021, 36, 2150004; Adv. Astron. (2021)2021; Eur. Phys. J. C 136(2021)503; Chin. J. Phys. 71(2021)365; Universe 7(2021)154.

[13] Harrison, E.R.: Rev. Mod. Phys. 39(1967)862.

[14] Gibbons, G.W.: Nucl. Phys. B 292(1987)784. 
[15] Barrow, J.D. and Yamamoto, K.: Phys. Rev. D 85(2012)083505.

[16] Böhmer, C.G., Hollenstein, L. and Lobo, F.S.N.: Phys. Rev. D 76(2007)084005; Goheer, N., Goswami, R. and Dunsby, P.K.S.: Class. Quantum Grav. 26(2009)105003; Seahra, S.S. and Böhmer, C.G.: Phys. Rev. D 79(2009)064009.

[17] Böhmer, C.G. and Lobo, F.S.N.: Phys. Rev. D 79(2009)067504.

[18] Li, J.T., Lee, C.C. and Geng, C.Q.: Eur. Phys. J. C 73(2013)2315.

[19] Huang, H., Wu, P. and Yu, H.: Phys. Rev. D 91(2015)023507.

[20] Böhmer, C.G., Tamanini, N. and Wright, M.: Phys. Rev. D 92(2015)124067.

[21] Shabani, H. and Ziaie, A.H.: Eur. Phys. J. C 77(2017)31.

[22] Sharif, M. and Ikram, A.: Eur. Phys. J. Plus 132(2017)526; Astrophys. Space Sci. 363(2018)178.

[23] Sharif, M. Waseem, A.: Eur. Phys. J. Plus 133(2018)160; Astrophys. Space Sci.364(2019)221.

[24] Sharif, M. and Saleem, S.: Mod. Phys. Lett A 35(2020)2050152; ibid. 2050222.

[25] Ade, P.A.R. et al.: Astron. Astrophys. 594(2016)A13.

[26] Ellis, G.F.R. and Maartens, R.: Class. Quantum Grav. 21(2004)223; Ellis, G.F.R., Murugan, J. and Tsagas, C.G.: Class. Quantum Grav. $\mathbf{2 1}(2004) 233$.

[27] Sharif, M. and Ikram, A.: Int. J. Mod. Phys. D 26(2017)1750084.

[28] Sharif, M. Waseem, A.: Mod. Phys. Lett. A 33(2018)1850216. 